\title{
Real-time comparative evaluation of bioMerieux VITEK MS versus Bruker Microflex MS, two matrix- assisted laser desorption-ionization time-of-flight mass spectrometry systems, for identification of clinically significant bacteria
}

Wafaa Jamal ${ }^{1,2^{*}}$, M John Albert $^{1}$ and Vincent $O$ Rotimi ${ }^{1}$

\begin{abstract}
Background: Matrix-assisted laser desorption-ionization time-of-flight mass spectrometry (MALDI-TOF MS) recently became available for the identification of bacteria in routine diagnostic laboratories. It is rapid and cost-effective and likely to replace phenotypic identification. This study was undertaken to compare two MALDI-TOF MS-based, Bruker Microflex MS (BMS) and VITEK MS (VMS) systems, for identification (ID) of clinically significant bacterial isolates. Clinically relevant broad diversity of bacterial isolates obtained during a 6-consecutive months of routine laboratory processing of clinical specimens were subjected to ID by the BMS and VMS in parallel with Vitek 2, a conventional phenotypic system (CPS). For the BMS, the isolates were tested in duplicates directly and after pretreatment. Identification was provided with accompanying scores according to manufacturers' instructions. With VMS, single deposits of the same sets of isolates were tested in duplicates directly on MALDI-plate. Results were interpreted according to the manufacturer's protocols. Discrepant results were resolved by 165 rRNA gene amplification and sequencing.
\end{abstract}

Results: A total of 806 pathogens comprising 507 Gram-negative bacilli (GNB), 16 Gram-negative cocci (GNC), 267 Gram-positive cocci (GPC), and 16 Gram-positive bacilli (GPB) were tested. BMS and VMS correctly identified isolates to genus and species levels (ID 97.3\% and 93.2\%, and 99.8\% and 99.0\%, respectively). Both systems as well as the CPS correctly identified the majority of the species in the family Enterobacteriaceae, Pseudomonas spp., and Acinetobacter baumannii. Turnaround time for identification by BMS and VMS was $<20$ min compared with 24-48 h by the CPS.

Conclusions: VMS performed slightly better than BMS with GPC ID, especially the Streptococcus spp. Some S. mitis isolates were identified as S. pneumoniae by BMS. BMS and VMS were rapid and proved to be consistently accurate for producing bacterial identification in a fraction of time it takes for identification by CPS.

Keywords: Evaluation, MALDI-TOF mass spectrometry, Clinical bacterial isolates

\footnotetext{
* Correspondence: wjamal@hsc.edu.kw

${ }^{1}$ Department of Microbiology, Faculty of Medicine, Kuwait University and Microbiology Unit, Mubarak Al-Kabir Hospital, Jabriya, Kuwait

2Department of Microbiology, Faculty of Medicine, Kuwait University, P.O. Box 24923, Safat 13110, Kuwait
} 


\section{Background}

The routine identification of bacterial isolates in the hospital clinical diagnostic microbiology laboratory is currently done by analysis of phenotypic characteristics such as growth on selective and non-selective media, colonial morphology, Gram-stain morphology and typical biochemical reactions. These methods are laborious, timeconsuming and require a relatively long turn-around time. Fast and cost-effective matrix-assisted laser desorption ionization-time of flight mass spectrometry (MALDI-TOF MS)-based systems have been developed essentially to replace the biochemical and other phenotypic systems for routine identification of bacteria. This is because it is now necessary to have a low-cost, fast and reproducible method for bacterial identification in the routine diagnostic laboratory.

Initially, MALDI-TOF MS technique was developed for industrial use to analyze and identify proteins. However, two decades later, Holland et al., [1] and Krishnamurthy and Ross [2] demonstrated that it could be used for rapid identification of bacterial whole-cell based on spectral pattern. But its potential for routine use in microbial identification in clinical microbiology laboratory was only demonstrated a few years ago [3-6]. This technique is based on the analysis of microbial proteins by an ionization process devoid of fragmentation by coordinated action of laser and small organic acids of the matrix. These are then separated on the basis of their mass-to-charge ratios, a process which results in a characteristic mass spectral profile. Identification of microbes is based on the comparison of the protein spectrum generated from intact whole microbial cells to a database of species-specific reference protein profiles. Protein analysis by MALDI-TOF MS does not require lengthy biochemical reactions, thereby making it a more rapid identification strategy than traditional routine methods $[5,6]$.

Currently, commercial and user-friendly instruments containing different algorithms for the identification of microbial protein mass spectrum pattern along with a database for thousands of reference bacteria are now available. These are Bruker Microflex MS (Bruker Daltonics, Bremen, Germany), VITEK MS IBB (bioMerieux, Marcy l'Etoile, France), VITEK MS RUO (formerly Saramis, bioMerieux) and Andromas system (Andromas SAS, Paris, France) $[7,8]$. Many laboratories have focused on analysis of specific bacterial genera using the MALDI-TOF MS technology [9-14]. Other studies have analyzed general groups of bacteria $[15,16]$, and yeasts $[17,18]$, or evaluated its use in the routine clinical laboratory for bacterial identification [3-6,19,20].

In the current study, we carried out a comparative evaluation of the performance of Bruker Microflex MS (BMS, Daltonics) versus VITEK MS (VMS, bioMerieux) based on an updated classifier and database, in parallel with routine phenotypic Vitek 2 system (CPS, bioMerieux) and molecular methods i.e. 16S rRNA amplification and sequencing.

\section{Methods}

\section{Bacterial isolates}

Consecutive, clinically relevant, a broad diversity of bacterial isolates were tested during a period of six consecutive months (January 2012 to June 2012) of routine laboratory processing of clinical specimens in the Microbiology Laboratory, Mubarak Al Kabir hospital, Kuwait. Duplicate isolates from the same patient were excluded. The 806 isolates included in the study encompassed Enterobacteriaceae, non-fermentative Gram-negative bacilli, other Gram-negative bacteria, staphylococci and related species, streptococci and related species, and Gram-positive rods. They were isolated from different sources- blood, urine, pus, tissue, wound, respiratory secretion and body fluid. All identification tests were carried out simultaneously by BMS, VMS and CPS as a part of routine diagnostic workup.

\section{Vitek 2 system}

Routine bacterial identification in the laboratory was carried out by Vitek 2 (bioMerieux). Gram-positive and Gram-negative isolates were inoculated into GP ID (card no. 21342) and GN ID (card no. 21341) respectively. Neisseria and Haemophilus species were identified by NH ID (card no. 21346). When necessary, complementary biochemical tests were done.

\section{MALDI-TOF Bruker MS (BMS)}

Measurements were done with a Microflex LT mass spectrometer (Bruker Daltonics) using FlexControl software (version 3.3). The spectra were imported into the integrated MALDI Biotyper software (version 3.0) and analyzed by standard pattern matching with a default setting. The isolates were tested in duplicates directly and after pretreatment. A colony from blood agar plate was directly spotted on the MALDI plate, and then overlaid with $1 \mu \mathrm{l}$ of matrix solution and air-dried. The loaded plate was then placed in the instrument according to the manufacturer's instructions. The spectrum of each isolate was compared with that in the database and the identification was provided by a score of reliability $(<1.7=$ ID not reliable; $\geq 1.7$ and $<2.0=$ ID at genus level; $\geq 2.0=$ ID at species level). A result was considered species consistent if all matches with a $\log$ score $\geq 2.0$ were of the same species and if matches with a log score between 2.0 and 1.7 correspond only to other species of the same genus. A genus consistent result was accepted if all $\log$ scores $\geq 1.7$ belong to the same genus. 


\section{MALDI TOF VITEK MS (VMS)}

The isolates were tested in duplicates directly on the MALDI-plate. A single bacterial colony was deposited on the target slide, followed by the addition of the matrix (VITEK MS-CHCA) and air drying. The loaded slide was inserted into the VITEK MS machine. VITEK mass spectrometer was used to generate spectra of the bacterial suspension and the Biotyper software (version 2.0) was used to analyze the results. Microbial identification was achieved by obtaining the spectra using MALDI-TOF technology and analyzing the spectra with the VITEK MS database. The peaks from these spectra were compared with the characteristic pattern for the species, genus or family of the microorganism, leading to identification of the organism. The results were evaluated according to a colored index: green for $\geq 90 \%$ identity, yellow for $85-89.9 \%$ identity and white for $<85 \%$ identity. All of the identifications to the genus or species level fell into the green zone, with a score of $>90 \%$ considered reliable. Scores between 85 and $90 \%$ were considered as acceptable identification. A cut-off of $90 \%$ was chosen for the VITEK MS.

\section{Quality control}

Escherichia coli ATCC 8739 was included as a positive control and matrix alone with no organism was included as a negative control in each run in both MALDI-TOF systems.

\section{Discrepancy}

Discrepancies were defined as different genus- or specieslevel identification obtained from the VITEK MS, Microflex Bruker MS or conventional method Vitek 2. Whenever there was a discrepancy, the analysis was repeated by both systems to eliminate the possibility of contamination. Thereafter, any other disagreements in the ID were resolved by performing $16 \mathrm{~S}$ rRNA gene amplification and sequencing on the discrepant isolates.

\section{S rRNA gene amplification}

DNA from colonies obtained from samples with discordant results emanating from the 3 identification systems was extracted as previously described by Boom et al. [21]. The 16S rRNA genes were amplified and sequenced using universal 16S rRNA-specific primers [22]. The sequences obtained were compared with sequences present in the GeneBank database using BLAST software (http://www. ncbi.nih.gov).

\section{Ethics statement}

The evaluation of diagnostic systems was performed in the routine Clinical Microbiology Laboratory whose job is to provide support for patient care. Ethical approval is not required for analysis of routine clinical specimens sent to this laboratory.

\section{Results}

A total of 806 clinically significant pathogens comprising 507 Gram-negative bacilli (GNB), 16 Gram-negative cocci (GNC), 267 Gram-positive cocci (GPC), and 16 Grampositive bacilli (GPB) made up of 39 genera and 70 species were tested during the study period, as shown in Tables 1 , 2, 3 and 4. Of the 806 isolates, conventional Vitek 2 system correctly identified 795 (98.6\%) to the genus level and $777(96.4 \%)$ to the species level. VITEK MS identified 805 (99.9\%) to the genus level and 798 (99.0\%) to the species level. Bruker Microflex MS identified 784 (97.3\%) and 751 (93.2\%) to the genus and species levels, respectively; 17 isolates were also correctly identified to the species level but at no reliable ID score of $<1.7$. Both MALDI-TOF systems took less than 20 minutes from colony to ID result compared with $\geq 24$ h by CPS.

As shown in Table 1, both VMS and Vitek 2 gave correct and acceptable identification for 504/507 (99.4\%) Gramnegative bacilli. Using $16 \mathrm{~S}$ rRNA sequencing results as the gold standard, two Providencia rettgeri were misidentified as Providencia stuartii and one Ralstonia solanacearum was identified as Ralstonia pickettii. BMS gave correct and an acceptable identification for 490/507 (96.6\%) Gramnegative bacilli. It could identify $11 \mathrm{Gram}$-negative bacilli (1 Klebsiella pneumoniae, 3 Acinetobacter baumannii, 1 Acinetobacter lwoffii, 1 Salmonella species, 1 Stenotrophomonas maltophilia, 1 P. rettgeri, 1 Burkholderia cepacia, and 2 Sphingomonas paucimobilis) correctly but with an unacceptable ID score of $<1.7$ when compared to sequencing results. However, the system misidentified 4 Gramnegative bacilli with an acceptable high ID score $>2.0$ ( $1 \mathrm{~A}$. baumannii as Acinetobacter genomospecies 13TU [this organism has been recently named as Acinetobacter nosocomialis, http://www.bacterio.net/], 2 Aeromonas hydrophila/ caviae as Aeromonas jandaei, and $1 R$. solanacearum as Ralstonia insidiosa) compared to sequencing results. Both systems correctly identified all Escherichia coli, Pseudomonas aeruginosa, Enterobacter spp., Proteus spp., Serratia spp., Citrobacter spp. and Morganella spp.

As shown in Table 2, Vitek 2 gave correct and acceptable identification for 255 (95.5\%) of the 267 Grampositive cocci, while it could not identify Rhodococcus equi as it is not present in its database. It misidentified 12 Gram-positive cocci: 1 Staphylococcus epidermidis misidentified as Staphylococcus capitis, 2 Micrococcus luteus/ lylae as Kocuria kristinae, 1 Streptococcus lutetiensis as Streptococcus infantarius, 8 Streptococcus mitis/oralis as Streptococcus constellatus; 3, Streptococcus peroris; 1, Streptococcus pluranimalium; 1, Streptococcus pentosaceus; 1, Streptococcus sanguis; 1, Streptococcus thoraltensis; 1. VMS correctly identified at an acceptable level, 263 (98.5\%) 
Table 1 Discrepancies and error in the conventional identification method (Vitek 2) and MALDI-TOF for the identification of 507 Gram-negative bacteria

\begin{tabular}{|c|c|c|c|c|c|c|c|}
\hline \multirow{3}{*}{$\begin{array}{l}\text { Final identification (no } \\
\text { of isolates) }\end{array}$} & \multicolumn{7}{|c|}{ No of isolates } \\
\hline & \multicolumn{2}{|c|}{$\begin{array}{l}\text { Conventional identification } \\
\text { method (Vitek 2) }\end{array}$} & \multicolumn{2}{|c|}{$\begin{array}{l}\text { VITEK MS } \\
\text { identification }\end{array}$} & \multicolumn{2}{|c|}{$\begin{array}{l}\text { Bruker MS } \\
\text { identification }\end{array}$} & \multirow{2}{*}{$\begin{array}{l}\text { 16S rRNA sequence result } \\
\text { (number of discrepant } \\
\text { organisms sequenced) }\end{array}$} \\
\hline & No ID & Mis-ID & No ID & Mis - ID & No ID & Mis-ID & \\
\hline Escherichia coli (181) & 0 & 0 & 0 & 0 & 0 & 0 & \\
\hline Klebsiella pneumoniae (71) & 0 & 0 & 0 & 0 & 0 & 1 (score $<1.7)$ & K. pneumoniae (1) \\
\hline Klebsiella oxytoca (2) & 0 & 0 & 0 & 0 & 0 & 0 & \\
\hline $\begin{array}{l}\text { Pseudomonas } \\
\text { aeruginosa (66) }\end{array}$ & 0 & 0 & 0 & 0 & 0 & 0 & \\
\hline $\begin{array}{l}\text { Acinetobacter } \\
\text { baumannii (39) }\end{array}$ & 0 & 0 & 0 & 0 & 0 & $\begin{array}{l}1 \text { (Acinetobacter } \\
\text { genomospecies 13TU)*, } \\
3 \text { (score }<1.7)\end{array}$ & A. baumannii (4) \\
\hline Acinetobacter Iwoffii (2) & 0 & 0 & 0 & 0 & 0 & 1 (score $<1.7)$ & A. Iwoffii (1) \\
\hline Salmonella spp. (29) & 0 & 0 & 0 & 0 & 0 & 1 (score $<1.7$ ) & Salmonella spp. (1) \\
\hline Enterobacter cloacae (14) & 0 & 0 & 0 & 0 & 0 & 0 & \\
\hline $\begin{array}{l}\text { Enterobacter } \\
\text { aerogenes ( } 7 \text { ) }\end{array}$ & 0 & 0 & 0 & 0 & 0 & 0 & \\
\hline Enterobacter asburiae (5) & 0 & 0 & 0 & 0 & 0 & 0 & \\
\hline Pantoea agglomerans (1) & 0 & 0 & 0 & 0 & 0 & 0 & \\
\hline Proteus mirabilis (17) & 0 & 0 & 0 & 0 & 0 & 0 & \\
\hline $\begin{array}{l}\text { Stenotrophomonas } \\
\text { maltophilia (18) }\end{array}$ & 0 & 0 & 0 & 0 & 0 & $1($ score $<1.7)$ & S. maltophilia (1) \\
\hline Serratia marcescens (15) & 0 & 0 & 0 & 0 & 0 & 0 & \\
\hline Citrobacter koseri (9) & 0 & 0 & 0 & 0 & 0 & 0 & \\
\hline Citrobacter freundii (1) & 0 & 0 & 0 & 0 & 0 & 0 & \\
\hline Morganella morganii (7) & 0 & 0 & 0 & 0 & 0 & 0 & \\
\hline Achromobacter xylosoxidans (5) & 0 & 0 & 0 & 0 & 0 & 2 (Acinetobacter spp.) & A. xylosoxidans (2) \\
\hline Providencia rettgeri (4) & 0 & $\begin{array}{l}2 \text { (Providencia } \\
\text { stuartii) }\end{array}$ & 0 & 2 (P. stuartii) & 0 & $1($ score $<1.7)$ & P. rettgeri (2) \\
\hline Burkholderia cepacia (2) & 0 & 0 & 0 & 0 & 0 & 1 (score $<1.7$ ) & B. cepacia (1) \\
\hline Sphingomonas paucimobilis (2) & 0 & 0 & 0 & 0 & 0 & 2 (score $<1.7$ ) & S. paucimobilis (2) \\
\hline $\begin{array}{l}\text { Aeromonas hydrophilal } \\
\text { caviae (2) }\end{array}$ & 0 & 0 & 0 & 0 & 0 & 2 (Aeromonas jandaei) & A. hydrophila/caviae (2) \\
\hline $\begin{array}{l}\text { Ralstonia } \\
\text { solanacearum (1) }\end{array}$ & 0 & $\begin{array}{l}1 \text { (Ralstonia } \\
\text { pickettii) }\end{array}$ & 0 & 1 (R. pickettii) & 0 & 1 (Ralstonia insidiosa) & R. solanacearum (1) \\
\hline $\begin{array}{l}\text { Chryseobacterium } \\
\text { indologenes (1) }\end{array}$ & 0 & 0 & 0 & 0 & 0 & 0 & \\
\hline Plesiomonas shigelloides (1) & 0 & 0 & 0 & 0 & 0 & 0 & \\
\hline Vibrio parahaemolyticus (1) & 0 & 0 & 0 & 0 & 0 & 0 & \\
\hline Campylobacter jejuni (1) & 0 & 0 & 0 & 0 & 0 & 0 & \\
\hline Bordetella pertussis (1) & 0 & 0 & 0 & 0 & 0 & 0 & \\
\hline Bordetella parapertussis (1) & 0 & 0 & 0 & 0 & 0 & 0 & \\
\hline Yersinia enterocolitica (1) & 0 & 0 & 0 & 0 & 0 & 0 & \\
\hline
\end{tabular}

No ID = no identification in spite of its presence in database; Mis-ID = misidentification; ${ }^{*}$ Current name is Acinetobacter nosocomialis.

of the 267 Gram-positive cocci; Rhodococcus equi was not present in its database but identified by $16 \mathrm{~S}$ rRNA sequencing. VMS misidentified 5 Gram-positive cocci: $1 S$. epidermidis as Staphylococcus capitis, 1 Staphylococcus warneri as Staphylococcus auricularis, 1 Staphylococcus lutetiensis as Staphylococcus infantarius, 1 S. mitis/oralis as 
Table 2 Discrepancies and error in the conventional identification method (Vitek 2) and MALDI-TOF for the identification of 267 Gram-positive bacteria

\begin{tabular}{|c|c|c|c|c|c|c|c|}
\hline \multirow{3}{*}{$\begin{array}{l}\text { Final identification } \\
\text { (no of isolates) }\end{array}$} & \multicolumn{7}{|c|}{ Number of isolates } \\
\hline & \multicolumn{2}{|c|}{$\begin{array}{l}\text { Conventional identification } \\
\text { method (Vitek 2) }\end{array}$} & \multicolumn{2}{|c|}{$\begin{array}{l}\text { VITEK MS } \\
\text { identification }\end{array}$} & \multicolumn{2}{|c|}{$\begin{array}{l}\text { Bruker MS } \\
\text { identification }\end{array}$} & \multirow{2}{*}{$\begin{array}{l}\text { 16S rRNA sequence } \\
\text { results (number of } \\
\text { discrepant organism } \\
\text { sequenced) }\end{array}$} \\
\hline & No ID & Mis-ID & No ID & Mis-ID & No ID & Mis-ID & \\
\hline $\begin{array}{l}\text { Staphylococcus } \\
\text { aureus (53) }\end{array}$ & 0 & 0 & 0 & 0 & 0 & 0 & \\
\hline $\begin{array}{l}\text { Staphylococcus } \\
\text { epidermidis (34) }\end{array}$ & 0 & 1 (Staphylococcus capitis) & 0 & 1 (S. capitis) & 0 & 0 & S. epidermidis (2) \\
\hline $\begin{array}{l}\text { Staphylococcus } \\
\text { haemolyticus (11) }\end{array}$ & 0 & 0 & 0 & 0 & 0 & 0 & \\
\hline S. capitis (11) & 0 & 0 & 0 & 0 & 0 & 0 & \\
\hline $\begin{array}{l}\text { Staphylococcus } \\
\text { hominis (7) }\end{array}$ & 0 & 0 & 0 & 0 & 0 & 0 & \\
\hline $\begin{array}{l}\text { Staphylococcus } \\
\text { lugdunensis (4) }\end{array}$ & 0 & 0 & 0 & 0 & 0 & 0 & \\
\hline $\begin{array}{l}\text { Staphylococcus } \\
\text { caprae (1) }\end{array}$ & 0 & 0 & 0 & 0 & 0 & 1 (Staphylococcus auricularis) & S. caprae (1) \\
\hline $\begin{array}{l}\text { Staphylococcus } \\
\text { warneri (2) }\end{array}$ & 0 & 0 & 0 & S.auricularis & 0 & 1 (S. epidermidis) & S. warneri (1) \\
\hline $\begin{array}{l}\text { Streptococcus } \\
\text { agalactiae (46) }\end{array}$ & 0 & 0 & 0 & $\begin{array}{l}1 \text { (Nocardia } \\
\text { asteroides) }\end{array}$ & 0 & $\begin{array}{l}1 \text { (Alloiococcus otitis), } 1 \\
\text { (Lactobacillus sakei) }\end{array}$ & S. agalactiae (3) \\
\hline $\begin{array}{l}\text { Rothia } \\
\text { mucilaginosa (1) }\end{array}$ & 0 & 0 & 0 & 0 & 0 & 0 & \\
\hline $\begin{array}{l}\text { Enterococcus } \\
\text { faecalis (29) }\end{array}$ & 0 & 0 & 0 & 0 & 0 & 0 & \\
\hline $\begin{array}{l}\text { Enterococcus } \\
\text { faecium (6) }\end{array}$ & 0 & 0 & 0 & 0 & 0 & 0 & \\
\hline $\begin{array}{l}\text { Enterococcus } \\
\text { raffinosus (3) }\end{array}$ & 0 & 0 & 0 & 0 & 0 & 0 & \\
\hline $\begin{array}{l}\text { Enterococcus } \\
\text { avium (1) }\end{array}$ & 0 & 0 & 0 & 0 & 0 & 0 & \\
\hline $\begin{array}{l}\text { Streptococcus } \\
\text { pyogenes (7) }\end{array}$ & 0 & 0 & 0 & 0 & 0 & 0 & \\
\hline $\begin{array}{l}\text { Micrococcus luteus/ } \\
\text { lylae (6) }\end{array}$ & 0 & 2 (Kocuria kristinae) & 0 & 0 & 0 & $\begin{array}{l}3 \text { (1 Clostridium beijeriuckii, } 2 \\
\text { Propionibacterium acnes) }\end{array}$ & M. luteus (5) \\
\hline $\begin{array}{l}\text { Rhodococcus } \\
\text { equi (1) }\end{array}$ & NA & NA & NA & NA & 0 & 0 & R. equi (1) \\
\hline $\begin{array}{l}\text { Streptococcus } \\
\text { parasanguis (3) }\end{array}$ & 0 & 0 & 0 & 0 & 0 & 1 (Alloiococcus otitis) & S. parasanguis (1) \\
\hline $\begin{array}{l}\text { Streptococcus } \\
\text { salivarius (1) }\end{array}$ & 0 & 0 & 0 & 0 & 0 & 0 & \\
\hline $\begin{array}{l}\text { Streptococcus } \\
\text { lutetiensis (1) }\end{array}$ & 0 & 1 (Streptococcus infantarius) & 0 & $\begin{array}{l}1 \text { (Streptococcus } \\
\text { infantarius) }\end{array}$ & 0 & 0 & S. lutetiensis (1) \\
\hline $\begin{array}{l}\text { Streptococcus } \\
\text { dysagalactiae (2) }\end{array}$ & 0 & 0 & 0 & 0 & 0 & 0 & \\
\hline $\begin{array}{l}\text { Streptococcus mitis/ } \\
\text { oralis (26) }\end{array}$ & 0 & $\begin{array}{l}3 \text { (Streptococcus constellatus), } \\
1 \text { (Streptococcus peroris), } \\
1 \text { (Streptococcus pluranimalium), } \\
1 \text { (Streptococcus pentosaceus), } \\
1 \text { (Streptococcus sanguis), } \\
1 \text { (Streptococcus thoraltensis) }\end{array}$ & 0 & 1 (S. constellatus) & 0 & $\begin{array}{l}18 \text { (Streptococcus } \\
\text { pneumoniae) }\end{array}$ & S. mitis / oralis (26) \\
\hline
\end{tabular}


Table 2 Discrepancies and error in the conventional identification method (Vitek 2) and MALDI-TOF for the identification of 267 Gram-positive bacteria (Continued)

\begin{tabular}{|c|c|c|c|c|c|c|c|}
\hline $\begin{array}{l}\text { Streptococcus } \\
\text { anginosus (2) }\end{array}$ & 0 & 0 & 0 & 0 & 0 & 0 & \\
\hline $\begin{array}{l}\text { Streptococcus } \\
\text { gallolyticus (1) }\end{array}$ & 0 & 0 & 0 & 0 & 0 & 1 (Propionibacterium acnes) & S. gallolyticus (1) \\
\hline $\begin{array}{l}\text { Granulicatella } \\
\text { adiacens (2) }\end{array}$ & 0 & 2 (S. sanguinis, S. thoraltensis) & 0 & 0 & 0 & $\begin{array}{l}2 \text { (S. pneumoniae, } \\
\text { Streptococcus oralis) }\end{array}$ & G. adiacens (2) \\
\hline S. pneumoniae (5) & 0 & 0 & 0 & 0 & 0 & 0 & \\
\hline $\begin{array}{l}\text { Streptococcus } \\
\text { mutans (1) }\end{array}$ & 0 & 0 & 0 & 0 & 0 & 1 (P. acnes) & S. mutans (1) \\
\hline
\end{tabular}

No ID = no identification in spite of its presence in database; Mis - ID = misidentification; NA = not present in database

S. constellatus; and 1 Streptococcus agalactiae as Nocardia asteroides. Out of 267 Gram-positive cocci, BMS gave correct and acceptable identification for 237 (88.8\%). It misidentified 30 Gram-positive cocci isolates. These were the following: 1 Staphylococcus caprae as S. auricularis, 1 Staphylococcus warneri as S. epidermidis, 2 S. agalactiae as Alloiococcus otitis and Lactobacillus sakei, 3 Micrococcus luteus/lylae as Clostridium beijeriuckii 1; and Propionibacterium acnes 2; 1 Streptococcus parasanguis as Alloiococcus otitis. Compared to sequencing results, 18 of $26 \mathrm{~S}$. mitis/ oralis group were misidentified as Streptococcus pneumoniae. In addition, $1 S$. gallolyticus was misidentified as P. acnes and 1 Streptococcus mutans as P. acnes. All the 3 systems correctly identified all Staphylococcus aureus, Streptococcus pyogenes, S. pneumoniae and Enterococcus spp. to the genus and species levels.

Table 3 shows the discrepancies in the conventional and MALDI-TOF methods (Vitek 2, BMS and VMS) for identification of 16 Gram-negative cocci. Vitek 2 identified all Gram-negative cocci to the genus and species levels except 5 Neisseria subflava for which database is absent. VMS identified 15 (93.8\%) of 16 Gram-negative cocci correctly, and misidentified 1 Neisseria cinerea as $N$. subflava, when compared to sequencing results. However, BMS correctly identified only 8 (50\%) of 16
Gram-negative cocci to the species and genus levels. It correctly identified 2 of 8 Haemophilus influenzae, but with an ID score of $<1.7$ and it misidentified $1 N$. cinerea as Neisseria sicca and $5 \mathrm{~N}$. subflava as Neisseria flava, when compared to sequencing results.

The discrepancies and errors among Vitek 2, BMS and VMS for identification of 16 Gram-positive bacilli are shown in Table 4 . Vitek 2 correctly identified only 4 of 16 Gram-positive bacilli. Corynebacterium terpenotabidum, Corynebacterium striatum, Lactobacillus jensenii and Lactobacillus rhamnosus are not present in Vitek 2 data base. Compared to sequencing results, Vitek 2 misidentified 1 Nocardia otitidiscaviarum as Listeria monocytogenes. VMS correctly identified to an acceptable level, 14 (87.5\%) of 16 Gram-positive bacilli. It misidentified 1 Corynebacterium terpenotabidum as Clostridium septicum and $1 N$. otitidiscaviarum as L. monocytogenes. BMS identified 13/16 (81.3\%) Gram-positive bacilli, but misidentified C. terpenotabidum as Clostridium beijerinckii, 1 Listeria ivanovii as L. monocytogenes and $1 N$. otitidiscaviarum as L. monocytogenes.

\section{Discussion and conclusions}

MALDI-TOF MS systems have been now implemented in many clinical microbiology laboratories because of

Table 3 Discrepancies and error in the conventional identification method (Vitek 2) and MALDI-TOF for the identification of 16 Gram-negative cocci

\begin{tabular}{|c|c|c|c|c|c|c|c|}
\hline \multirow{3}{*}{$\begin{array}{l}\text { Final identification } \\
\text { (no of isolates) }\end{array}$} & \multicolumn{7}{|c|}{ Number of isolates } \\
\hline & \multicolumn{2}{|c|}{$\begin{array}{l}\text { Conventional identification } \\
\text { method (Vitek 2) }\end{array}$} & \multicolumn{2}{|c|}{$\begin{array}{l}\text { VITEK MS } \\
\text { identification }\end{array}$} & \multicolumn{2}{|c|}{$\begin{array}{l}\text { Bruker MS } \\
\text { identification }\end{array}$} & \multirow{2}{*}{$\begin{array}{l}\text { 16S rRNA sequence results } \\
\text { (number of discrepant } \\
\text { organisms sequenced) }\end{array}$} \\
\hline & No ID & Mis - ID & No ID & Mis - ID & No ID & Mis - ID & \\
\hline Haemophilus influenzae (8) & 0 & 0 & 0 & 0 & 0 & 2 (score <1.7) & H. influenzae (2) \\
\hline Neisseria cinerea (1) & 0 & 0 & 0 & 1 (Neisseria subflava) & 0 & 1 (Neisseria sicca) & N. cinerea (1) \\
\hline Neisseria subflava (5) & NA & NA & 0 & 0 & 0 & Neisseria flava (5) & N. subflava (5) \\
\hline Neisseria meningitidis (1) & 0 & 0 & 0 & 0 & 0 & 0 & \\
\hline Moraxella catarrhalis (1) & 0 & 0 & 0 & 0 & 0 & 0 & \\
\hline
\end{tabular}

No ID = no identification in spite of its presence in database; Mis - ID = misidentification; NA = not present in database. 
Table 4 Discrepancies and error in the conventional identification method (Vitek 2) and MALDI-TOF for the identification of 16 Gram-positive bacilli

\begin{tabular}{|c|c|c|c|c|c|c|c|}
\hline \multirow{3}{*}{$\begin{array}{l}\text { Final identification (no of } \\
\text { isolates) }\end{array}$} & \multicolumn{7}{|c|}{ Number of isolates } \\
\hline & \multicolumn{2}{|c|}{$\begin{array}{l}\text { Conventional identification } \\
\text { method (Vitek 2) }\end{array}$} & \multicolumn{2}{|c|}{ VITEK MS identification } & \multicolumn{2}{|c|}{ Bruker MS identification } & \multirow{2}{*}{$\begin{array}{l}\text { 16S rRNA sequence results } \\
\text { (number of discrepant } \\
\text { organisms sequenced) }\end{array}$} \\
\hline & No ID & Mis - ID & $\begin{array}{l}\text { No } \\
\text { ID }\end{array}$ & Mis - ID & $\begin{array}{l}\text { No } \\
\text { ID }\end{array}$ & Mis - ID & \\
\hline $\begin{array}{l}\text { Corynebacterium } \\
\text { terpenotabidum (1) }\end{array}$ & NA & NA & 0 & $\begin{array}{l}1 \text { (Clostridium } \\
\text { septicum) }\end{array}$ & 0 & $\begin{array}{l}1 \text { (Clostridium } \\
\text { beijerinckii) }\end{array}$ & C. terpenotabidum (1) \\
\hline Lactobacillus jensenii (3) & NA & NA & 0 & 0 & 0 & 0 & L. jensenii (3) \\
\hline Corynebacterium striatum (5) & NA & NA & 0 & 0 & 0 & 0 & C. striatum (5) \\
\hline Lactobacillus rhamnosus (2) & NA & NA & 0 & 0 & 0 & 0 & L. rhamnosus (2) \\
\hline Erysipelothrix rhusiopathiae (1) & 0 & 0 & 0 & 0 & 0 & 0 & \\
\hline Listeria monocytogenes (1) & 0 & 0 & 0 & 0 & 0 & 0 & \\
\hline Listeria ivanovi (2) & 0 & 0 & 0 & 0 & 0 & 1 (L. monocytogenes) & L. ivanovii (1) \\
\hline Nocardia otitidiscaviarum (1) & 0 & 1 (L. monocytogenes) & 0 & $\begin{array}{l}1 \text { (L. } \\
\text { monocytogenes) }\end{array}$ & 0 & 1 (L. monocytogenes) & N. otitidiscaviarum (1) \\
\hline
\end{tabular}

No ID = no identification spite of its presence in database; Mis - ID = misidentification; NA = not present in database.

their efficiency, cost-effectiveness and rapid identification of different bacterial isolates. This technology is dependent on the reference strains included in their databases. Thus, when an isolate is tested, the species of the reference strain with the closest match is used for identification of the tested strain.

An important advantage of MALDI-TOF system is rapid identification of bacteria in just under 10 min compared to CPS that takes 16-20 h. The commercial systems allow the rapid identification of $S$. aureus but MALDI-TOF can recognize different coagulase negative staphylococci (CoNS). Identification of the exact species of CoNS may be very useful because some CoNS can cause serious infections and it may help to differentiate contaminants from true infections caused by CoNS [23]. A recent comparative study by Dupont et al., [24] in which MALDI-TOF MS and Vitek 2 were evaluated for the identification of 234 CoNS representing 20 different species showed that MALDI-TOF MS significantly performed better than Vitek 2 (93.2\% versus $75.2 \%$ ) with fewer misidentifications. In our study, all the $53 \mathrm{~S}$. aureus isolates were correctly identified by MALDI-TOF MS and Vitek 2, while only $1 \mathrm{~S}$. epidermidis was misidentified by Vitek 2 and 2 isolates each were misidentified by BMS and VMS.

S. pneumoniae is a major human pathogen and it has to be identified reliably and accurately in the clinical microbiology laboratory. In our hands, the CPS and both MALD-TOF systems identified it correctly which is discordant with the study of Seng et al. [5], which reported that about $50 \%$ of $S$. pneumoniae isolates were misidentified as Streptococcus parasanguinis, a member of Streptococcus mitis group. However, it should be pointed out that in our study BMS and VMS misidentified 18 of 26 and 1 of $26 \mathrm{~S}$. mitis/oralis group as S. pneumoniae, respectively, a finding that may clinically impact patient management. Similar misidentification has been reported by others in which MALDI-TOF MS system was found to misidentify S. mitis/oralis as S. pneumoniae [6,25]. This misidentification may be explained by the fact that $S$. pneumoniae is strikingly similar to $S$. mitis genetically [26] and that the resolving power of MALDI-TOF is not sufficient to discriminate between the two species [18]. Therefore, supplementary tests such as optochin disk susceptibility and bile solubility are needed for the correct identification of $S$. pneumoniae. It is important to subject an isolate to these supplementary tests prior to reporting it as S. pneumoniae because insertion of additional spectra of these species into the BMS database may not solve the problem. In contrast to our findings, another study did not misidentify any of the 369 non-pneumococcal streptococci and related genera as S. pneumoniae [27]. Therefore, MALDI-TOF MS analysis cannot be used alone for identification of these organisms in all laboratories. It must be pointed out that all enterococci isolates were correctly identified to the species level by both MALDI-TOF MS systems which is concordant with the report by van Veen et al., [6].

An important similarity in the performance of both MALDI-TOF MS and Vitek 2 was their ability to correctly identify Gram-negative bacteria, particularly the Enterobacteriaceae with few exceptions to genus and species levels. Compared to the CPS identification of nonfermenting Gram-negative bacilli which is frequently protracted, expensive and difficult, these rapid systems appear to be superior. The explanation for the problems sometimes encountered with the CPS may be attributed to the mucoid nature of some of these organisms or the fact that they are inert biochemically [28]. Some previous studies reported misidentification of S. maltophilia as Pseudomonas 
beteli, and Pseudomonas geniculata or Pseudomonas hibiscicola by BMS. This is thought to be related to a high level of homology between the genera and the species $[5,6]$. However, all our $18 \mathrm{~S}$. maltophilia were correctly identified by both BMS and VMS although 1 isolate which was identified by BMS had a low ID score of $<1.7$.

In this study, the Corynebacterium species collected in our routine practice were not identified by Vitek 2 primarily because of a lack of database for this species. Only 1 out of 6 isolates was misidentified as Clostridium species by both BMS and VMS. This may be explained by a lack of species diversity as previously alluded to by Dubois et al. [27]. In the past, Gram-positive bacilli were not routinely identified to species level as are Gramnegative rods isolated from clinical specimens. The use of MALDI-TOF MS in the clinical microbiology laboratory will provide species-level identification of these bacteria. Conceivably, identification of Gram-positive bacilli to species level may allow us to learn more about the real clinical significance of these organisms and perhaps help determine their hither - to underappreciated pathogenic potential.

An important limitation of this study was the relatively small number of Gram-negative cocci and Grampositive bacilli encountered during this study which makes a general evaluation regarding their identification rather weak. A prospective evaluation study involving a large number of stock clinical isolates of these groups is underway.

Several studies have also assessed the use of MALDITOF MS to detect the mechanisms of antibiotic resistance in Enterobacteriaceae, such as identification of $\beta$-lactams and $\beta$-lactam modified/degraded products $[29,30]$, detection of resistance proteins within the cell $[31,32]$ and detection of mutations within resistance genes through mini-sequencing [33]. In addition, MALDI-TOF MS has been used in clinical practice to detect Enterobacteriaceae producing extended spectrum $\beta$-lactamase from positive blood cultures [34]. However, the limitation of these reports is that these mechanisms will detect only one type of resistance mechanism to certain antibiotics e.g. $\beta$-lactams, which makes the clinical implication of their findings unsure at this time. Another notable limitation of the rapid MALDI-TOF MS systems is that neither of them could perform susceptibility testing for all antibiotics. In effect, the physician still has to wait for another $24 \mathrm{~h}$ to make an informed and definitive decision on appropriate antibiotic therapy.

In conclusion, both MALDI-TOF MS systems have the potential to replace the conventional methods for identification of the majority of pathogens isolated in the clinical microbiology laboratory. MALDI-TOF is a rapid, simple, inexpensive, user-friendly and high-throughput proteomic technique for identification of clinically significant bacteria (except viridans streptococci) which can be implemented in a routine, conventional, laboratory setting.

\section{Competing interests}

WJ and VOR are members of the Regional Advisory Board of Astellas.

\section{Authors' contributions}

WJ made substantial contributions to conception and design, acquisition and analysis of data and drafted the manuscript. MJA was involved in 165 rRNA sequencing and revision of the manuscript for important intellectual content. VOR made substantial contributions to the conception and design, and revision of the manuscript critically for important intellectual content. All authors read and approved the final manuscript.

\section{Acknowledgments}

We thank Mrs. Rola Saleem for her technical work.

Received: 24 June 2014 Accepted: 7 November 2014

Published online: 30 November 2014

\section{References}

1. Holland RD, Wilkes JG, Rafii F, Sutherland JB, Person CC, Voorhees KJ, Lay JO Jr: Rapid identification of intact whole bacteria based on spectral pattern using matrix-assisted laser desorption/ionization mass spectrometry. Rapid Commun Mass Spectrom 1996, 10:1227-1232.

2. Krishnamurthy T, Ross PL: Rapid identification of bacteria by direct matrixassisted laser desorption ionization mass spectrometric analysis of whole cells. Rapid Commun Mass Spectrom 1996, 10:1992-1996.

3. Bizzini A, Durussel C, Bille J, Greub G, Prod'hom G: Performance of matrixassisted laser desorption ionization-time of flight mass spectrometry for identification of bacterial strains routinely isolated in a clinical microbiology laboratory. J Clin Microbiol 2010, 48:1549-1554.

4. Cherkaoui A, Hibbs J, Emonet S, Tangomo M, Girard M, Francois P, Schrenzel J: Comparison of two matrix-assisted laser desorption ionization-time of flight mass spectrometry methods with conventional phenotypic identification for routine identification of bacteria to the species level. J Clin Microbiol 2010, 48:1169-1175.

5. Seng P, Drancourt M, Gouriet F, La Scola B, Fournier PE, Rolain JM, Raoult D: Ongoing revolution in bacteriology: routine identification of bacteria by matrix-assisted laser desorption ionization time-of-flight mass spectrometry. Clin Infect Dis 2009, 49:543-551.

6. van Veen SQ, Claas EC, Kuijper EJ: High-throughput identification of bacteria and yeast by matrix-assisted laser desorption ionization-time of flight mass spectrometry in conventional medical microbiology laboratories. J Clin Microbiol 2010, 48:900-907.

7. Emonet S, Shah NH, Cherkaoui A, Schrenzel J: Application and use of various mass spectrometry methods in clinical microbiology. Clin Microbiol Infect 2010, 16:1604-1613.

8. Sauer S, Kliem M: Mass spectrometry tools for the classification and identification of bacteria. Nat Rev Microbiol 2010, 8:74-82.

9. Dubois D, Leyssene D, Chacornac JP, Kostrzewa M, Schmit PO, Talon R, Bonnet R, Delmas J: Identification of a variety of Saphylococcus species by matrix-assisted laser desorption ionization-time of flight mass spectrometry. J Clin Microbiol 2010, 48:941-945.

10. Pennanec $X$, Dufour $A$, Haras $D$, Rehel $K$ : A quick and easy method to identify bacteria by matrix-assisted laser desorption/ionization time-offlight mass spectrometry. Rapid Commun Mass Spectrom 2010, 24:384-392.

11. Seibold E, Maier T, Kostrzewa M, Zeman E, Splettstoesser W: Identification of Francisella tularensis by whole-cell matrix-assisted laser desorption ionization-time of flight mass spectrometry: fast, reliable, robust, and cost-effective differentiation on species and subspecies levels. J Clin Microbiol 2010, 48:1061-1069.

12. Stephan R, Ziegler D, Pfluger V, Vogel G, Lehner A: Rapid genus- and species-specific identification of Cronobacter spp. by matrix-assisted laser desorption ionization-time of mass spectrometry. J Clin Microbiol 2010, 48:2846-2851.

13. Szabados F, Woloszyn J, Richter C, Kaase M, Gatermann S: Identification of molecularly defined Staphylococcus aureus strains using matrix-assisted laser desorption/ionization time of flight mass spectrometry and the Biotyper 2.0 database. J Med Microbiol 2010, 59:787-790. 
14. Vargha M, Takats Z, Konopka A, Nakatsu CH: Optimization of MALDI-TOF MS for strain level differentiation of Arthrobacter isolates. J Microbio Methods 2006, 66:399-409.

15. Degand N, Carbonnelle B, Dauphin B, Beretti JL, Le Bourgeois M, Semet-Gaudelus I, Segonds C, Berche P, Nassif X, Ferroni A: Matrix-assisted laser desorption ionization- time of flight mass spectrometry for identification of nonfermenting gram-negative bacilli isolated from cystic fibrosis patients. J Clin Microbiol 2008, 46:3361-3367.

16. Mellmann A, Cloud J, Maier T, Keckevoet U, Ramminger I, Iwen P, Dunn J, Hall G, Wilson D, Lasala P, Kostrzewa M, Harmsen D: Evaluation of matrix-assisted laser desorption ionization-time-of-flight mass spectrometry in comparison to $16 \mathrm{~S}$ rRNA sequencing for species identification of nonfermenting bacteria. J Clin Microbiol 2008, 46:1946-1954.

17. Marklein G, Josten M, Klanke U, Muller E, Horre R, Maier T, Wenzel T, Kostrzewa M, Bierbaum G, Hoerauf A, Sahl HG: Matrix-assisted laser desorption ionization-time of flight mass spectrometry for fast and reliable identification of clinical yeast isolates. J Clin Microbiol 2009, 47:2912-2917

18. Stevenson LG, Drake SK, Shea YR, Zelazny AM, Murray PR: Evaluation of matrix-assisted laser desorption ionization-time of flight mass spectrometry for identification of clinically important yeast species. J Clin Microbiol 2010, 48:3482-3486

19. Bessede E, Angla-Gre M, Delagarde Y, Sep Hieng S, Menard A, Megraud F: Matrix-assisted laser-desorption/ionization BIOTYPER: experience in the routine of a University hospital. Clin Microbiol Infect 2011, 17:533-538.

20. Jamal WY, Shahin M, Rotimi VO: Comparison of two matrix-assisted laser desorption/ ionization-time of flight (MALDI-TOF) mass spectrometry methods and API 20 AN for identification of clinically relevant anaerobic bacteria. J Med Microbiol 2013, 62:540-544.

21. Boom R, Sol CJ, Salimans MM, Jansen CL, Wertheim-van Dillen PM, van der Noordaa J: Rapid and simple method for purification of nucleic acids. J Clin Microbiol 1990, 28:495-503.

22. Hiraishi A: Direct automatic sequencing at $16 \mathrm{~S}$ rDNA amplified by polymerase chain reaction from bacterial cultures without DNA purification. Lett Appl Microbiol 1992, 15:210-213.

23. von Eiff C, Peters G, Heilmann C: Pathogenesis of infections due to coagulase-negative staphylococci. Lancet Infect Dis 2002, 2:677-685.

24. Dupont C, Sivadon-Tardy V, Bille E, Dauphin B, Beretti JL, Alvarez AS, Degand N, Ferroni A, Rottman M, Herrmann JL, Nassif X, Ronco E, Carbonnelle E: Identification of clinical coagulase-negative staphylococci, isolated in Microbiology laboratories, by matrix-assisted laser desorption/ionizationtime of flight mass spectrometry and two automated systems. Clin Microbiol Infect 2010, 16:998-1004.

25. Karpanoja P, Harju I, Rantakokko-Jalava K, Haanpera M, Sarkkinen H: Evaluation of two matrix-assisted laser desorption ionization-time of flight mass spectrometry systems for identification of viridans group streptococci. Eur J Clin Microbiol Infect Dis 2014, 33:779-788.

26. Kilian M, Poulsen K, Blomqvist T, Havarstein LS, Bek-Thomsen M, Tettelin H, Sorensen UB: Evolution of Streptococcus pneumoniae and its close commensal relatives. PLoS One 2008, 3:e2683.

27. Dubois D, Segonds C, Prere M-F, Marty N, Oswald E: Identification of clinical Streptococcus pneumoniae isolates among other alpha and nonhemolytic streptococci by use of the VITEK MS matrix-assisted laser desorption ionization-time of flight mass spectrometry system. J Clin Microbiol 2013, 51:1861-1867.

28. Bosshard PP, Zbinden R, Abels S, Boddinghaus B, Altwegg M, Bottger EC 16S rRNA gene sequencing versus the API 20 NE system and the VITEK 2 ID-GNB card for identification of non-fermenting Gram-negative bacteria in the clinical laboratory. J Clin Microbiol 2006, 44:1359-1366.

29. Burckhardt I, Zimmermann S: Using matrix-assisted laser desorption ionization-time of flight mass spectrometry to detect carbapenem resistance within 1 to 2.5 hours. J Clin Microbiol 2011, 49:3321-3324.

30. Hrabak J, Studentova V, Walkova R, Zemlickova H, Jakubu V, Chudackova E, Gniadkowski M, Pfeifer Y, Perry JD, Wilkinson K, Bergerova T: Detection of NDM-1, VIM-1, KPC, OXA-48, and OXA-162 carbapenemases by matrix-assisted laser desorption ionization-time of flight mass spectrometry. J Clin Microbio/ 2012, 50:2441-2443.

31. Cai JC, Hu YY, Zhang R, Zhou HW, Chen GX: Detection of OmpK36 porin loss in Klebsiella spp. by matrix-assisted laser desorption ionization-time of flight mass spectrometry. J Clin Microbiol 2012, 50:2179-2182.
32. Schaumann R, Knoop N, Genzel GH, Losensky K, Rosenkranz C, Stingu CS Schellenberger W, Rodloff AC, Eschrich K: A step towards the discrimination of $\beta$-lactamase-producing clinical isolates of Enterobacteriaceae and Pseudomonas aeruginosa by MALDI-TOF mass spectrometry. Med Sci Monit 2012, 18:MT71-MT77.

33. Ikryannikova LN, Shitikov EA, Zhivankova DG, I'ina EN, Edelstein MV, Govorun VM: A MALDI TOF MS-based minisequensing method for rapid detection of TEM-type extended spectrum- $\beta$-lactamases in clinical strains of Enterobacteriaceae. J Microbiol Methods 2008, 75:385-391.

34. Oviano M, Fernandez B, Fernandez A, Barba MJ, Mourino C, Bou G: Rapid detection of enterobacteriaeae producing extended spectrum $\beta$ lactamases directly from positive blood cultures by matrix-assisted laser desorption ionization -time of flight mass spectrometry. Clin Microbiol Infect 2014, doi:10.1111/1469-0691.12729 [Epub ahead of print].

\section{Submit your next manuscript to BioMed Central and take full advantage of:}

- Convenient online submission

- Thorough peer review

- No space constraints or color figure charges

- Immediate publication on acceptance

- Inclusion in PubMed, CAS, Scopus and Google Scholar

- Research which is freely available for redistribution
C) Biomed Central 